\title{
Transformation based Approach of Combining Iris and Fingerprint Biometric at Confidence Level
}

\author{
S. M. Rajbhoj \\ Ph.D. Research Scholar \\ BVUCOE, Bharati VidyapeethUniversity, Pune, \\ India
}

\begin{abstract}
Unimodal Biometric systems depending on information from single trait has many limitations. These are noisy input, inability to enroll into system, unacceptable error rates, spoofing and universality of traits. Multibiometric systems is likely to enhance the recognition accuracy by integration the evidence presented by multiple sources of information. In this paper a multibiometric system using transformation based fusion of two most used biometric traits, fingerprint and iris at confidence level is proposed. Features are extracted from individual biometric modalities by efficient algorithm. These features are first matched with their corresponding templates to compute the corresponding match scores. Match scores obtained from different traits are then transformed using different techniques and combined by simple sum rule to generate a fused match score. The proposed framework is evaluated using standard database. This system overcomes limitation of unimodal biometric system and gives improved performance accuracy. An equal error rate achieved by this system is 0.400 . The benefit of this approach is that, it does not require any estimation as in density based approach or a large number of training score as in classifier based approach. Image or feature level fusion is expected to result in better performance, but this approach outperforms feature level as well as decision level fusion of iris and fingerprint.
\end{abstract}

\section{General Terms}

Recognition, Algorithms, fingerprint, iris.

\section{Keywords}

Biometric,unimodal, multibiometric, match score, confidence level, fusion, ROC.

\section{INTRODUCTION}

Biometry or Biometric recognition is human identification or verification system based on physiological and/or behavioral characteristics (traits) of an individual. This system can authorize or establish an individual's identity based on "who he/she is", rather than by "what he/she has" or "what he/she remembers". Few of the trait linked with an entities are fingerprint, face, iris, hand geometry, signature, voice, palm print, retina, ear, hand vein, voice pattern, odor or the DNA information [1]. Unimodal biometric system performs person recognition based on single source of biometric information. These systems have to deal with of number of limitations such as intra-class variations, inter-class similarities, spoof attacks and universality of trait. Apart from these limitation, noisy data of defective sensors, the high failure to enroll rate and failure to capture rate and scalability are other problems concerned with these systems. Multimodal biometric system seeks to overcome some of these limitations by integrating the characteristics presented by multiple biometric information sources. It combines information from two or more biometric sources in a single system. It may combine information from multiple biometric traits, multiple sensors, multiple

\author{
P. B. Mane \\ Principal, AISSMS, Institute of \\ Information Technology Pune, \\ India
}

representations, multiple snapshots or matching algorithms [2].

In this paper we present a transformation based approach of combining iris and fingerprint biometric at confidence level in a multibiometric system. Next section gives review of past work, a brief framework of proposed multibiometric system is presented in section 3. In Section 4 performance evaluationis presented and discussed. Section 5 gives the conclusion.

\section{REVIEW OF PAST WORK}

A wide range of information integration methods are proposed in biometric literature.

They are broadly classified depending on levels at which the information is fused, type of fusion strategy and techniques used. The traits information can be combined at decision level, rank level, score level, feature level and sensor or image level.

Several traits were fused at score level to improve performance accuracy in multimodal biometrics.

Brunelli and Falavigna [3] use a HyperBF network to combine voice and face matchers.

Verlinde and Cholet [4] compared the performance accuracy of three different classifiers, k-Nearest Neighbor classifier, the decision tree classifier, and a logistic regression model based classifier while combining the match scores from three biometric matchers.

L. Hong and A. K. Jain [5] integrated face and fingerprint traits of an individual for person identification at match score level. The score were first modified by normalization technique before fusing to improve accuracy.

A hand based verification system that combines the geometric features of the hand with palm prints at the feature and match score levels is described by Kumar et al. [6]. Their results showed that match score level fusion is far better than feature level fusion.

Sanderson and Paliwal [7] use a support vector machine (SVM) to fuse the scores of face and speech experts. They designed SVM classifiers based on a modified Bayesian classifier and a piece-wise linear classifier.

Face and iris traits of an individual were fused by Y. Wang et al.[8] using simple un-weighted, weighted sum rule neural network with radial basis function (RBFNN) and Fisher's discriminant analysis.

Both iris and fingerprint are highly accurate biometric identifiers. But some users are not able to give his proper iris image due to ambient light, handling of devices, problematic iris scanners, eye diseases or absence of that trait. Fingerprint recognition system can also have problem if scars and cuts are present on fingertip or sensor is incapable of capturing fingerprint images of certain individual due to various reasons. Although there has been much research on 
combining different biometrics for a variety of purposes, there is less consideration on the combining finger print and iris.Baig et al. [9] made use of single hamming distance matcher for fusing fingerprint and iris. Conte et al. [10] combined feature level information by frequency based approach and Lumini and Nanni [11] presented a case study using SVM. Here we have attempted to combine these two identifiersiris and fingerprint, by transformation based method at confidence level.

\section{THE PROPOSED MULTIBIOMETRIC SYSTEM FRAMEWORK}

The proposed framework consists of fingerprint match score extraction module, iris match score extraction module, score transformation and fusion module. Fingerprint match score extraction module consists of fingerprint preprocessing, primarily enhancement and resizing of fingerprint image and minutiae extraction. In iris score extraction module first the iris region is segmented from eye image and then it is normalized. Iris feature are than extracted by Haar wavelet transform. Fig 1 shows the block diagram of proposed framework.

\subsection{Fingerprint Score Extraction Module}

The input for this module is fingerprint image. The fingerprint image is first pre-processed. Preprocessing mainly consists of image enhancement. Fingerprint enhancement algorithm applies a set of intermediate steps on the input image. Firstly input image is normalized to a pre-specific mean and variance. Normalization reduces the variations in grey level values along ridges and furrows, which facilitates the succeeding processing steps.

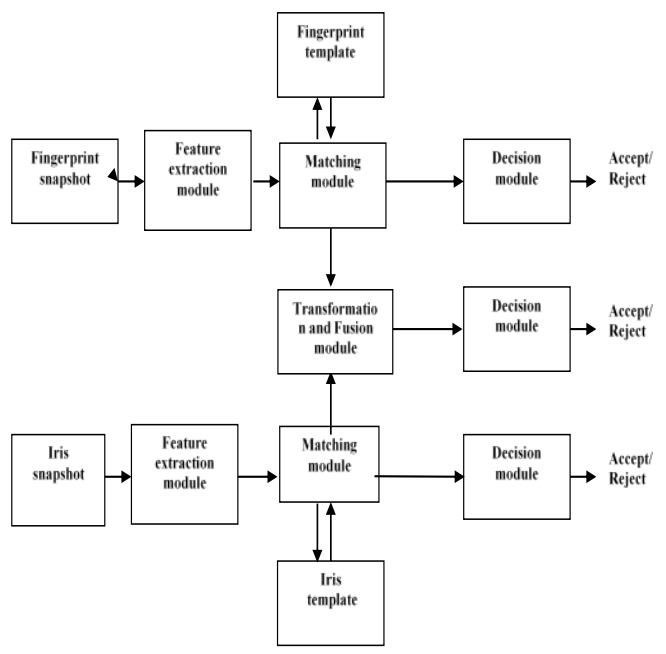

Fig 1: Block Diagram of Proposed Multibiometric system

Then local ridge orientation is calculated which defines invariant coordinates for ridges and furrows. In local neighborhood where there is no minutiae points, the grey levels along ridges and furrows is modeled as a sinusoidalshaped wave along a direction normal to the local ridge orientation. This is done to estimate the ridge frequency image and to remove undesired noise and preserve the true ridge and furrow structures, Gabor filters are used for it [12]. Gabor filters have both frequency selective and orientation selective properties. Output of filtering gives the enhanced image.

Local adaptive thresholding method is used to binarize the image. Here 8-bit Gray image is transformed into a 1-bit image with 0 for ridges and 1 for valleys. Ridges in the fingerprint are emphasized with black color while valleys by white. In this locally adaptive technique first the image is divided into blocks of $32 \times 32$ pixels and pixel value is set to 1 if its value is larger than the mean intensity value of the current block to which the pixel belongs.

Elimination the redundant pixels of ridges till the ridges are just one pixel wide is then carried out. This is done using the morphological thinning function

Minutiae are extracted from the thinned image [13].All minutiae are categorized by its $(\mathrm{x}, \mathrm{y})$ location in the image, and the orientation $\theta$ of the ridge on which it is detected. Minutiae marking are now done using templates for each $3 \mathrm{x}$ 3 pixel window. If the central pixel is 1 and has exactly 3 onevalue neighbor's, then this pixel represents a bifurcation and if the central pixel is 1 and has only 1 one-value neighbor, then it is a termination. Output of various steps is shown in fig 2

The process of fingerprint matching involves comparing the feature vectors representing minutiae points, from the template stored and query image. The feature vector is described by different attributes. The feature vectors in database are arranged in order and then ordered vectors are compared with feature vector of query image by finding Euclidean distance between them. The sum of the Euclidean distances is the output of the matching process that indicates the similarity of two minutiae set that are matched. This summation of the Euclidean distances is the match score of fingerprint.

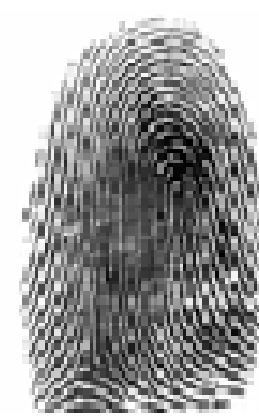

Input image

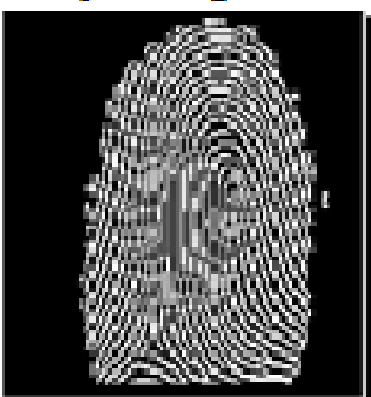

Enhanced Image

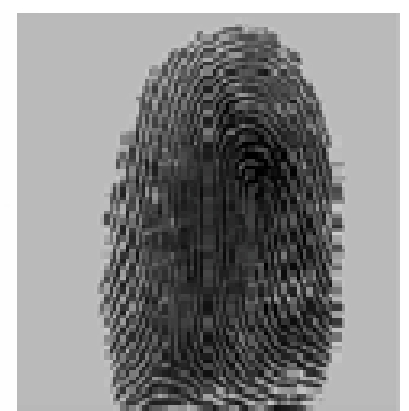

Equalized Image

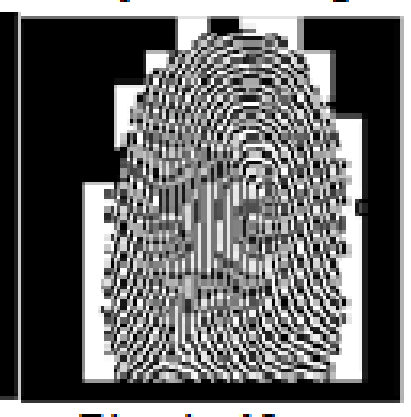

Binarized Image
Fig 2: Output of various steps of fingerprint algorithm

\subsection{Iris Score Extraction Module}

The iris is first extracted from image of an eye. The main steps of iris score extraction module are iris segmentation or localization, normalization and discrete wavelet transform. Segmentation determines the boundary of iris and pupil and removes eyelids and eyelashes. Integro-differential operator [14] is used to detect the iris and pupil contour.

The integro- differential operator is represented as 


$$
\begin{aligned}
& \max (r, x p, y 0) \mid G S \sigma(r) \\
& * \frac{\partial}{\partial r} \oint_{r, x 0, y 0} \frac{A(x, y)}{2 \Pi r} d s \mid
\end{aligned}
$$

$A(x, y)$ is the eye image,

$r$ is the radius of the search,

\section{$G S \sigma(r)$ is a Gaussian smoothing function}

$s$ is the contour of the circle given by $x, y$ and $r$.

This ID operator searches for the boundary path having maximum variation in pixel values, by changing the values of radius and center $\mathrm{x}$ and $\mathrm{y}$ positions of the contour. The operator is applied in an iterative manner with the amount of smoothing gradually reduced in order achieve correct segmentation. This step gives the center co-ordinates and radius of iris and pupil. Linear Hough transform is used to remove eyelids and eyelashes if present.

Next stage is to transform the iris region into fixed dimensions. Daugman's rubber sheet model [15] is used for it. In case of pupil and iris are non-concentric, remapping formula is used to rescale points depending on the angle around the circle. This is given as follows.

$$
\begin{gathered}
r^{\prime}=\sqrt{(\alpha \beta)} \pm \sqrt{\left(\alpha \beta^{2}-\alpha-a^{2}\right)} \\
\alpha=X^{2}+\mathrm{Y}^{2},
\end{gathered}
$$

$\beta=\cos \left(\pi-\arctan \left(\frac{Y}{X}\right)-\theta\right)$

The displacement of the center of the pupil with respect to the center of the iris is given by $X, Y$ and $r$ is the distance between the edge of the pupil and the edge of the iris at angle $\theta$ and " $a$ " is the radius of the iris. Fig. 3 shows the output.

Wavelets have the advantage over traditional Fourier transform and can be used to decompose the data of the iris region into constituents that appear at different resolutions.

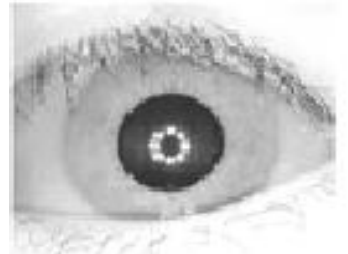

Input Image

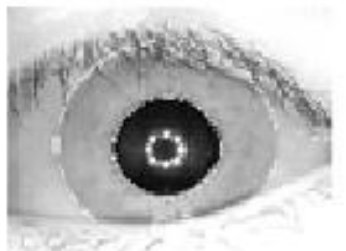

Iris Segmentation

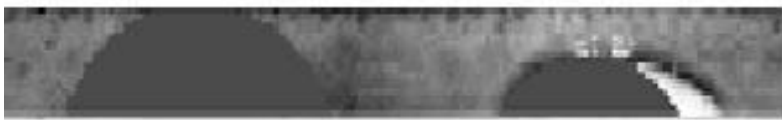

Iris Normalization

Fig 3: Output of segmentation and normalization steps of iris algorithm

Number of wavelet filters or bank of wavelets, is applied to the two dimensional iris region, one for each resolution [16]. The output is then encoded in order to provide a compact and discriminating representation of the iris pattern. Here Haar wavelet transform is used to extract features from the iris region. De-composing images with wavelet transform yields a multi-resolution images in each level. If $\mathrm{N} \mathrm{x} \mathrm{M}$ image is taken then it is decomposed up to $\mathrm{K}$ th level where $\mathrm{K}=1,2,3$ etc. The quadrants (sub-images) within the image indicated as HL, $\mathrm{LH}$ and $\mathrm{HH}$ represent detailed images for vertical, horizontal and diagonal orientation, correspondingly in the level one. The image LL represents to an approximation image that is further decomposed, resulting in level two wavelet decomposition. The result of the level three decomposition generates 64 sub images from which local texture information energy measure is obtained.

The energy measure is given as

$$
I E i=\sum_{j, k} S i(j, k)^{2}
$$

IEi energy measure for sub image Si, Energy measure is calculated for all 64 sub images. Wavelet energies of each iris sub image is used to calculate threshold.

The threshold [Th] is given as

$$
T h=K \cdot \frac{\vartheta(I E 1, \ldots \ldots \ldots \ldots I E n)}{\operatorname{Max}(I E 1, \ldots \ldots \ldots \ldots I E n)}
$$

Where $\mathrm{K}$ is constant,IE1...IEn are appropriate wavelet sub image energies of $1 \ldots . n$ sub images and $\mu$ (IE1........IEn) are mean wavelet peak energy values. After finding wavelet energies of sub images and threshold, iris code [17] is calculated as follows

$$
\begin{aligned}
& \text { Iris Code }(j)=1 \text { if } E j / E n>T \\
& \operatorname{IrisCode}(j)=0 \text { otherwise }
\end{aligned}
$$

Where $\mathrm{j}=0 \ldots . .63$. This results in 64 bits iris code.

The Iris Code derived from this process is compared with previously processed and stored Iris Code. This process of pattern matching evaluates the goodness of match between the acquired iris pattern and the candidate's data base entry. Based on this final decision is taken whether acquired data does or doesn't come from the same iris. Hamming distance is used as the distance measure which is given as

$$
H D=1 / B \sum_{i=1}^{B} V i[E X O R] W i
$$

Where Vi and Wi are query and database iris codes and B number of bits. These hamming distances are the match scores of Iris.

\subsection{Transformation of Score and Fusion Module}

The match scores which are the output of fingerprint and iris biometric modules are combined to generate a new match score that can be subsequently used for verification or identification. Fusion at this level is called confidence level fusion as there is confidence in processing match scores (compared with the processing raw biometric data or the feature set extracted from the data). Score obtained from above two modules may be non-homogeneous, may be have different distribution or have different ranges hence it is 
necessary to transform location and scale parameter of these score distribution into common domain.

Here we have used following transformation techniques [18] to transform both fingerprint and iris scores.

These are minmax, decimal, Zscore, median and median absolute deviation and tanh estimators.

Given matching scores $\left\{s_{k}\right\}, \mathrm{k}=1,2$,

[1 is fingerprint and 2 is Iris] then transformed scores are mathematically given as follows:

Min-max

$$
s^{\prime}=\frac{s-\min \left\{s_{k}\right\}}{\max \left\{s_{k}\right\}-\min \left\{s_{k}\right\}}
$$

Decimal scaling:

$$
\begin{gathered}
s^{\prime}=\frac{s}{10^{n}} \\
\mathrm{n}=\log _{10} \max \left\{s_{k}\right\}
\end{gathered}
$$

Z-score:

$$
s^{\prime}=\frac{s-\text { ? }}{\sigma}
$$

Median and Median Absolute Deviation (MAD):

$$
\begin{gathered}
s^{\prime}=\frac{s-\text { median }}{M A D} \\
\text { MAD }=\text { median }\left(\mid\left\{\mathrm{s}_{k}\right\}-\text { median } \mid\right)
\end{gathered}
$$

Tanh estimators

$$
s^{\prime}=0.5\left(\tanh \left(0.01 \frac{\left(s-?_{G H}\right)}{\sigma_{G H}}\right)+1\right)
$$

Here $\mu$ is mean, $\sigma$ is variance and GH is Gaussian distribution.

After score transformation we combine or fuse transformed scores of iris and fingerprint using simple sum rule. The output of the fusion module will then be given to decision module which uses this new matching score to determine an identity.

\section{PERFORMANCE EVALUATION}

The fingerprint database used for our experimentation is FVC 2004 database [19]. We have takeneight fingerprint images per subject for a total of forty subjects to have a database of 320 images. For iris database we have used the CASIA v4 database [20] which was obtained from Institute of Automation, Chinese Academy of Sciences. We have selected iris images of forty subjects and taken eight images of each subject. As both these databases are independent hence each fingerprint was assigned a corresponding iris image. The database thus formed had fingerprint and iris data from 40 subjects each having eight fingerprint images and eight iris images. The proposed fingerprint and iris algorithm were implemented in MATLAB. Each template vector is matched with every feature vector of the database. This results in 2560 mate subject and 99840 non mate subjects. The scores of the mate subjects are genuine scores and scores of non-mate subjects are imposter scores. Thus we get 2560 genuine scores and 99840 imposter scores. Thus fingerprint and iris score were obtained. These score were further transformed and fused. The accuracy of any biometric system is usually measured in terms of error rates i.e. FAR and FRR. GAR is used to measure the overall accuracy. The overall performance is brought together in by plotting receiver operating characteristics (ROC). ROC plot is a plot of false accept rate (FAR) and genuine accept rate (GAR) for all apparent thresholds, each point on the curve corresponds to particular threshold.

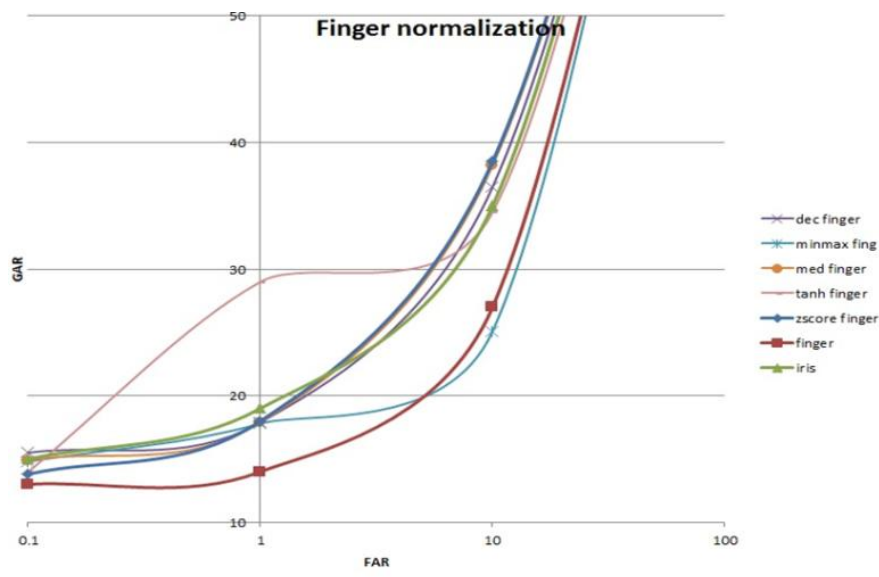

Fig.4. Receiver Operating Characteristics plot GAR v/s FAR).

Fig. 4 shows plot of FAR and GAR when score of fingerprint is transformed and iris score are not transformed and fusion is carried by simple sum rule. A corresponding plot of false accept rate (FAR) and false reject accept rate (FRR) is shown in Fig. 5.

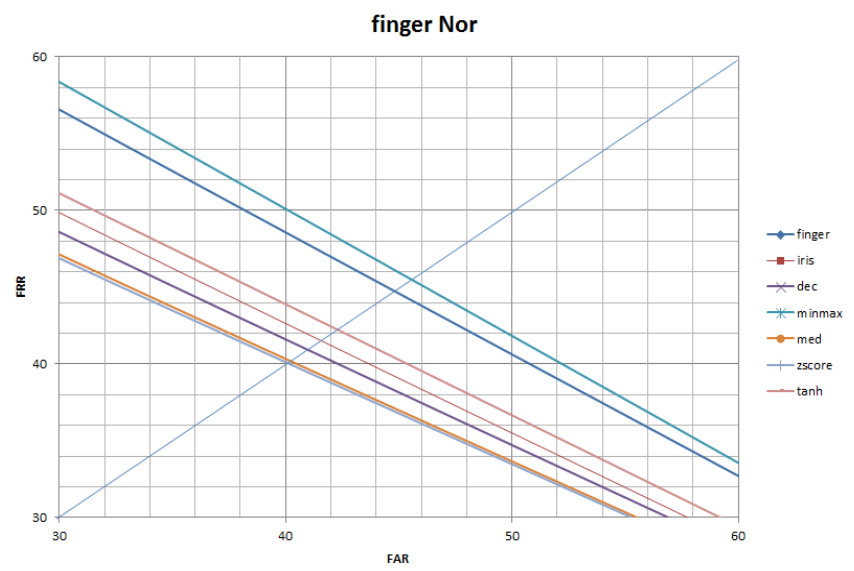

Fig.5. Receiver Operating Characteristics plot (FRR v/s FAR).

Table 1 shows EER values for different transformation techniques when only fingerprint scores are transformed and fused with untransformed iris scores and only iris scores are transformed and fused with untransformed fingerprint scores. The EER for unimodal fingerprint and iris systems are as follows Fingerprint EER=0.450 and Iris EER=0.416. From the table 1 we can say that we can achieve better results in terms of EER using this transformation based fusion approachof iris and fingerprint. Transforming fingerprint score gives better EER for decimal, Zscore and Tanh techniques while minmax and mean techniques works better for transforming iris scores.

Zscore transformation technique gives best result[EER=0.400] as fingerprint score has a Gaussian distribution while in iris minmax technique gives an EER of 0.405 as the range of iris score distribution is less. Transformation of score by these 
techniques gives far more good accuracy than the most accurate biometric iris in this case [EER=0.416]. We can also see from ROC in Fig 4 tanh gives good GAR in midrange compared to all other techniques. These framework provide EER of 0.400 which is better than (EER of 1.447) given by fusion of fingerprint and iris using SVM [11] and (EER=9 and 2.36) given by fusion of fingerprint and iris at feature level [9][10].

Table 1. EER for different Transformation methods placed above the table

\begin{tabular}{|l|l|l|l|l|l|}
\hline $\begin{array}{l}\text { Transformat } \\
\text { ion methods }\end{array}$ & $\begin{array}{l}\text { Decim } \\
\text { al }\end{array}$ & $\begin{array}{l}\text { Minm } \\
\text { ax }\end{array}$ & Mean & Zscore & Tanh \\
\hline $\begin{array}{l}\text { Only } \\
\text { Fingerprint }\end{array}$ & 0.410 & 0.456 & 0.402 & 0.400 & 0.422 \\
\hline Only Iris & 0.438 & 0.405 & 0.410 & 0.407 & 0.425 \\
\hline
\end{tabular}

\section{CONCLUSION}

The interest in multibiometrics has increased due to increasing importance of security, limitation of biometric systems and number of spoof attacks. In this paper, a multibiometric system using fingerprint and iris modality is presented where the scores of individual traits are first extracted by efficient algorithms. Scores are first transformed and combined using simple sum techniques at confidence level. The proposed framework is presented as option to using multiple verification systems in high security applications addressing the issues of non-universality, spoof attacks and limitation of unimodal systems. The result confirms that transformation based fusion of fingerprint and iris at confidence level gives better result compared to fusion at feature level, as features of iris and fingerprint at image level are incompatible and nonhomogeneous. Practical test shows simple sum is the most accurate fusion method. Zscore and min max techniques followed by sum fusion rule outperform other transformation techniques and transformation of fingerprint score by tanh technique enhance genuine acceptance rate in mid FAR range on this database. The advantage of this approach is estimation of score is not required and the amount of database required is less as no training is involved.The performance of system depends on type of fusion technique used hence future work will focus on using user specific matching threshold and user specific weights in fusion and obtaining quality scores.

\section{REFERENCES}

[1] A. K. Jain, A. Ross, and S. Prabhakar, "An introduction to biometric recognition," IEEE Trans. on Circuits and Systems for Video Technology, vol. 14, pp. 4-20, Jan 2004.

[2] A. Ross and A. K. Jain, "Information fusion in biometrics,"Pattern Recognition Letters, vol. 24, pp. 2115-2125, Sep 2003.

[3] Brunelli, R., Falavigna, D “ Person Identification using multiple cues." IEEE Trans. Pattern Analysis and Mach. Intell.17, 955-966 (1995)

[4] Verlinde, P., Cholet, G, “ Comparing decision fusion paradigms using k-NN based classifiers, decision trees and logistic regression in a multi-modal identity verification application", In: Proceedings of Second International Conference on Audio- andVideo-Based Biometric Person Authentication (AVBPA), pp.188-193. Washington D.C., USA (1999)

[5] L. Hong and A. K. Jain, "Integrating faces and fingerprints for personal identification," IEEE
Transactions on PAMI, vol. 20, pp. 1295-1307, Dec 1998.

[6] A. Kumar, D. C. M. Wong, H. C. Shen, and A. K. Jain, "Personal verification using palmprint and hand geometry biometric," in Proc. of 4th Int'l Conf. on Audio and Video-based Biometric Person Authentication (AVBPA), (Guildford, UK), pp. 668-678, Jun 2003.

[7] Sanderson, C., Paliwal, K.K “ Information Fusion and Person Verification Using Speech and Face Information". Research Paper IDIAP-RR 02-33, IDIAP (2002)

[8] Y. Wang, T. Tan, and A. K. Jain. "Combining Face and Iris Biometrics for Identity Verification.", In Fourth International Conference on Audio- and Video-based Biometric Person Authentication (AVBPA), Guildford, UK, pages 805-813, June 2003

[9] Asim Baig, Ahmed Bouridane, Fattih K., Gang Qu, "Fingerprint-Iris Fusion based Identification System using a Single Hamming Distance Matcher.",International Journal of Bio-Science and BioTechnology, Vol 1, No. 1, Dec 2009.

[10] V. Conte,C. Militello, F Sorbello, “A Frequency based approach for Feature Fusion in Fingerprint and Iris Multimodal Biometric Identification Systems", IEEE Transactions of System, Man and Cybernetics, vol-40, No.4, July2010.

[11] Lumini and Nanni, "When Fingerprint are combined with Iris - A Case Study: FVC2004 and CASIA “" International Journal of Network Security, Vol. 4 No. 1, pp27-34,Jan 2007

[12] L. Hong, Y. Wan, \& A.K. Jain, Fingerprint Image Enhancement: Algorithm and Performance Evaluation, IEEE Transactions on Pattern Analysis and Machine Intelligence, 20(8), 1998, 777-789

[13] S. M Rajbhoj and P. B. Mane,“An Improved binarization based algorithm using minutiae approach for Fingerprint Identification" International Journal of Engineering and Advanced Technology (IJEAT) Vol-1, Issue-6, August 2012

[14] J. G. Daugman, "How Iris Recognition Works", In IEEE Transactions on circuits and systems for video technology, vol. 14, no. 1,January 2004

[15] Libor Masek, "Recognition of Human Iris Patterns for Biometric Identification", Thesis Report School of Computer Science and Software Engineering, Western Australia, 2003

[16] Rossani F., Eslava M.T., Ea T., Aml F., Amara A., "Iris Identification and robustness evaluation of wavelet packetsbased algorithm", IEEE International Conference on image processing, vol.3, pp. III -257-260

[17] S. M. Rajbhoj and P. B. Mane, "Haar Wavelet Approach of Iris Texture Extraction for personal Recognition", International Journal of Innovative Technology and Exploring Engineering, Vol. 3 Issue 2, July - 2013

[18] A. K. Jain, K. Nandakumar, and A. Ross. Score Normalization in Multimodal Biometric Systems. Pattern Recognition, 38(12):2270\{2285, December 2005.

[19] Fingerprint verification competition. http://bias.csr.unibo.it/fvc2004

[20] CASIA Iris Image Database http://www.cbsr.ia.ac.cn/irisdatabase 